\title{
Performance Analysis of Stationary and Deterministic AODV Model
}

https://doi.org/10.3991/ijim.v14i17.16643

Kothai $\mathrm{G}^{(凶)}$, Poovammal E

SRM Institute of Science and Technology, Kattangulathur, Tamil Nadu

kothaigesrmist.edu.in

\begin{abstract}
Vehicular Adhoc Network (VANET) is an emerging technology that provides a digital communication among vehicles, persons and Road-Side Units (RSU). VANETs are highly vulnerable to cyber-attacks. These cyberattacks make a wrong illusion on traffic jam, can inject false information regarding traffics and injects large amount of spam messages that disrupts the normal functionalities. The main objective of the research work is to implement and analyze the different models that help in improving the traffic management. The scenarios are simulated, and the performance is analyzed using the OMNET++ Simulator.
\end{abstract}

Keywords Roadside Units, Cyberattacks, illusion, VANET, spam.

\section{$1 \quad$ Introduction}

Vehicular Ad-hoc Network (VANET) is one of the new research challenges in road traffic safety, traffic engineering or efficiency, comfort and quality of road travel, dynamic topology, frequent disconnection, etc [R1]. It helps in providing safety management, traffic management, driver comfort management, maintenance management. It also helps in maintaining communication among the group of vehicles that form a network. In VANET, communication takes place in two ways. When a vehicle directly communicates with other vehicle then it is $\mathrm{V} 2 \mathrm{~V}$ communication or Inter-Vehicular communication [R1, R15]. If a vehicle communicates with the infrastructure or roadside unit (RSU) then it is V2I communication [R6]. Some of the vehicular applications are Road Safety, Traffic Efficiency and Management, Comfort and Infotainment etc [R2].

Three basic components of system model are:

- Trusted Authority (TA)

- Road-Side Unit (RSU)

- On-Board Unit (OBU) 


\subsection{Trusted Authority}

TA is an authorized centre that provides registration and certification for RSU and OBU. It generates group key and signature that are further sent to the RSU in the domain [R3].

Road-Side Unit: Vehicles are managed and get communicated with other vehicle by RSU within a communication range. It also acts as a bridge between trusted authority and on-board unit through wired or wireless channel [R3].

On-Board Unit: OBU periodically broadcasts the traffic-related information such as location, speed, direction to improve the road environment for drivers and passengers. To store security information, each vehicle has a temper-proof device (TPD) [R3].

To improve the road safety and road efficiency, there is various communication standards available, which provide the radio access required for the communication between the vehicles [R4]. Emergency and warning messages, Interpersonal messages, Routing and safety messages and Information and Entertainment messages are the four types of messages that help in communicating between the vehicles and infrastructure in VANET [R4]. The basic standards for wireless access in VANETs are cellular access in vehicular environment $(2 \mathrm{G} / 2.5 \mathrm{G} / 3 \mathrm{G} / 4 \mathrm{G})$, dedicated short range communication (DSRC), Wireless access in vehicular Environment (WAVE) and WIMAX. The various attacks in VANET are Denial of Service attack (DOS), Distributed Denial of Service attack (DDOS), Sybil attack, Blackhole attack, Grey hole attack, worn hole attack, etc [R5]. VANET uses diverse cryptographical algorithms such as MD5, ECC, RSA, SHA1. There are some issues associated with these security protocols [R12]. Some routing protocols like AODV helps in detecting and preventing the attacks in VANET. The performances are compared between different routing protocols such as AODV, DSR and DSDV. It is observed that AODV performs better than other routing protocols in different scenarios like saving bandwidth and power consumption [R13, R14].

\section{AODV and Mobility Models}

The protocols, agents and other models are provided by the open source model library called INET framework for OMNET++ simulation environment [R11]. The Adhoc On-Demand Distance Vector (AODV) routing protocol is one of the reactive protocols where routes are created on demand [R9]. Instead of maintaining up-to-date route information, the AODV routing protocol broadcasts the messages over the network for route discovery process [R3]. This model undergoes Route Discovery and Route Maintenance. Route Discovery is done through Route REQUEST (RREQ) and Route REPLY (RREP) and Route Maintenance is done through Route ERROR (RERR).

When a source node wants to send a message to the destination node at first it checks in the Route Table and if the route exists then the packet is delivered through the route [R3]. If the route does not exist, then Source node broadcasts Route Request (RREQ) to the intermediate nodes. The broadcast Route Request consists of Source 
Address, Request ID, Source Sequence Number, Destination Address, Destination Sequence Number and Hop Count [R4, R10].

When the intermediate node receives the RREQ message it has two possibilities. If the node does not have a Valid Route to destination, then it forwards the request message to all other nodes [R4]. If the node has a valid route, then it Unicast the Route Reply (RREP) message. This RREP message contains Source Address, Destination Address, Destination Sequence Number, Hop Count and Lifetime [R4]. When the route request message is received multiple times, the duplicate copies will be discarded, by comparing the Broadcast ID and Source ID pairs.

The previous nodes broadcast ID is stored when RREQ is forwarded. If the Route Reply is not received before time expires, the entry will be deleted [R4]. All the nodes in the network will monitor the neighbourhood nodes and if any active route gets lost then it will send the Route Error Message (RERR) to all other nodes in the network [R4]. The active module called simple modules and grouping of simple modules called compound module are the two types of modules that helps in communicating with message passing [R7]. The position and orientation are described by mobility models in a 3D Euclidean coordinate system [R8]. The two different mobility models are single or group mobility models [R8]. The mobility model that describes the motion of entities independent to each other is known as single mobility model. A node undergoing motion and the members of the group are dependent to each other is provided by group mobility model [R8].

The AODV model is categorized as Stationary model and Mobility model. The Stationary model is classified as Stationary Mobility, Static Grid Mobility, Static Concentric Mobility. The Classification of Mobility model is shown in the below figure 1. 


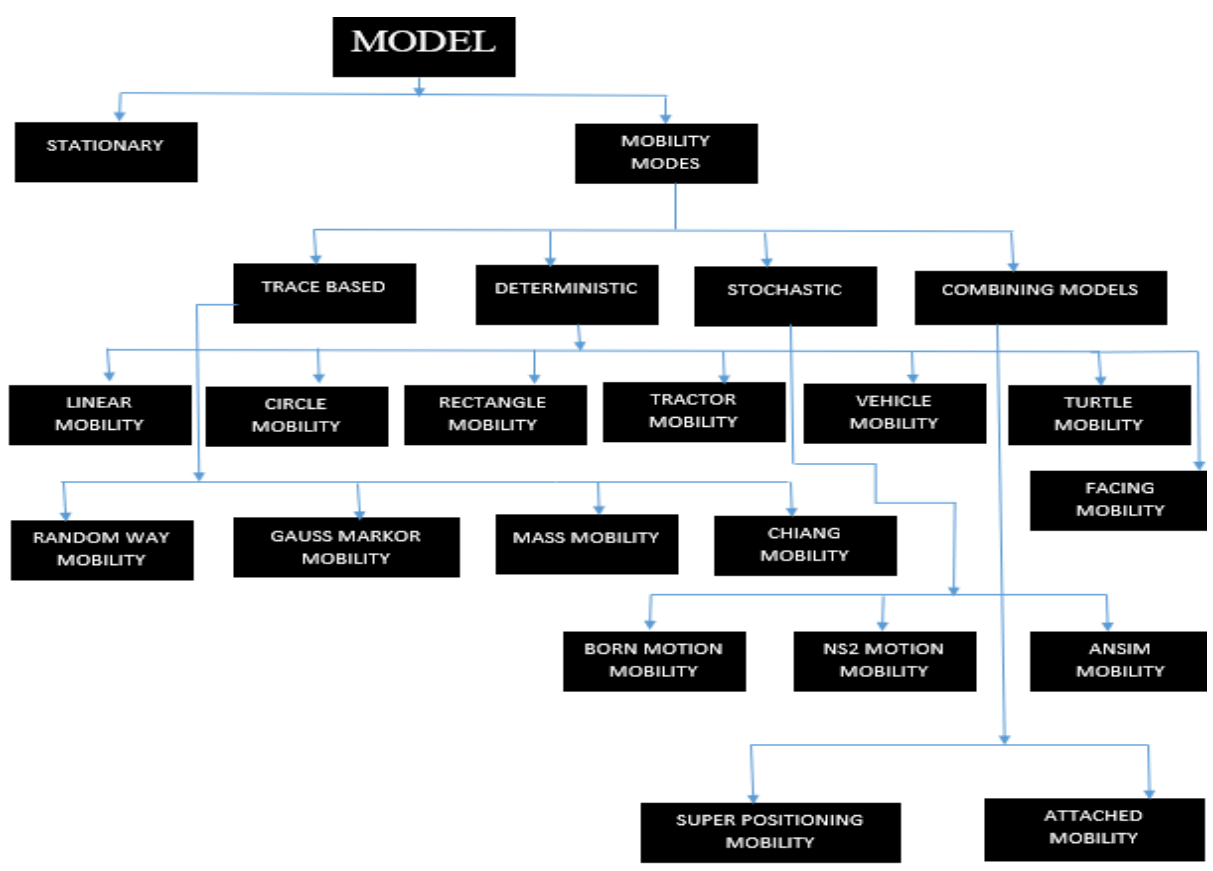

Fig. 1. Classification of Mobility Models

\section{Analysis of Results}

The simulation output of the AODV Stationary model and AODV Mobility model for fixed number of nodes as 20. The experiment run for 12 seconds and the calculated values based on the node shut down and start up time is tabulated are tabulated.

\subsection{Stationary model}

The Stationary models can define only the position and orientation but not the motion [R8]. The different stationary models are Stationary Mobility model that provides the positioning of nodes in deterministic and random manner [R8]. In a rectangular grid if mobility models are placed then it is Static Grid Mobility Model and if several models are placed in a set of Concentric Circles then it is Static Concentric Mobility [R8]. 
Paper-Performance Analysis of Stationary and Deterministic AODV Model

Table 1: Calculated values for Stationary model

\begin{tabular}{|c|c|c|c|c|}
\hline \multirow{2}{*}{ No of Events } & \multicolumn{4}{|c|}{ No of Nodes 20 } \\
\cline { 2 - 5 } & \multicolumn{4}{|c|}{ Time Taken When } \\
\cline { 2 - 5 } & 1 Node Shuts Down & $\begin{array}{c}\text { 5 Nodes Shuts } \\
\text { Down }\end{array}$ & $\begin{array}{c}\text { 10 Nodes Shuts } \\
\text { Down }\end{array}$ & $\begin{array}{c}\text { 15 Nodes Shuts } \\
\text { Down }\end{array}$ \\
\hline 200 & 3.19 & 3.19 & 3.19 & 3.19 \\
\hline 400 & 3.19 & 3.19 & 3.19 & 3.2 \\
\hline 600 & 3.2 & 3.2 & 3.2 & 3.2 \\
\hline 800 & 3.2 & 4.19 & 4.19 & 4.19 \\
\hline 1000 & 5.19 & 6 & 6.19 & 8.19 \\
\hline 1200 & 6.19 & 7.19 & 9.19 & 10.19 \\
\hline 1400 & 8.19 & 9.19 & 10.19 & 12 \\
\hline 1538 & 8.5 & 10.19 & 11.19 & \\
\hline 1600 & 9.19 & 11 & 12 & \\
\hline 1678 & 10 & 11.19 & & \\
\hline 1713 & 10.5 & 12 & & \\
\hline 1800 & 11.19 & & & \\
\hline 1924 & 12 & & & \\
\hline
\end{tabular}

Stationary Model

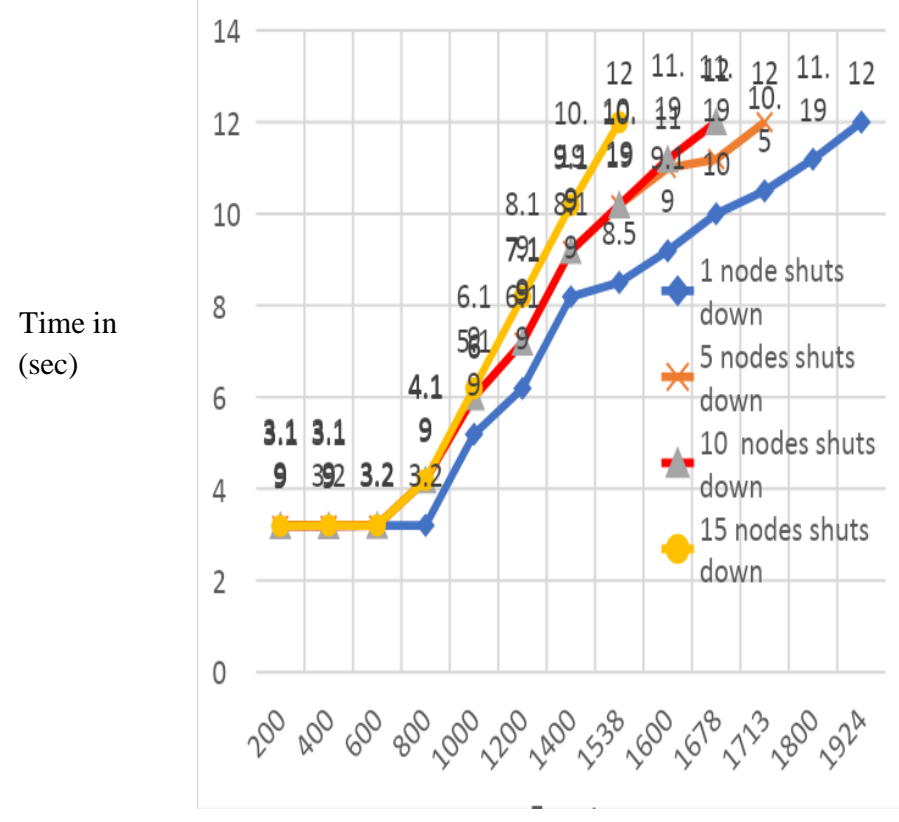

No. of Events

Fig. 2. Stationary Model

The Table I shows that when the nodes in a network are in stationary model, there are 1924 events occurred by 12 seconds even after one node shutdown from the 
network. If five nodes shutdown then total number of events is 1713 . If the ten and fifteen nodes shutdown then total number of events are 1678 and 1538. In a network when there is increase in number of nodes shutdown then total number of events gets decreases in this model.

\subsection{Deterministic models}

Deterministic mobility models are models that help in describing the motion using non-random mathematical models. The various deterministic mobility models are Linear Mobility helps in moving the nodes with constant speed and acceleration in linear manner. If the nodes move around circular and rectangular that is parallel to $\mathrm{XY}$ plane with constant speed, then it is Circle Mobility and rectangle mobility model. If the node orients towards the position of any other mobility model, then it is facing mobility model [R8].

Linear mobility model: The above Table II shows that when the nodes in a network are in linear mobility model there are 8523 events occurs by 12 seconds even after one node shutdown in the network. If five nodes shutdown then total number of events is 6780. If the ten and fifteen nodes shutdown then total number of events are 6354 and 5589. In a network when there is increase in number of nodes shutdown then total number of events gradually gets decreases in this model.

Table 2: Calculated values for Linear Deterministic Model

\begin{tabular}{|c|c|c|c|c|}
\hline \multirow{2}{*}{ No of Events } & \multicolumn{4}{|c|}{ No of Nodes 20 } \\
\cline { 2 - 5 } & \multicolumn{4}{|c|}{ Time Taken When } \\
\cline { 2 - 5 } & 1 Node Shuts Down & $\begin{array}{c}\text { 5 Nodes Shuts } \\
\text { Down }\end{array}$ & $\begin{array}{c}\text { 10 Nodes Shuts } \\
\text { Down }\end{array}$ & $\begin{array}{c}\text { 15 Nodes Shuts } \\
\text { Down }\end{array}$ \\
\hline 1000 & 3.19 & 3.19 & 3.19 & 3.19 \\
\hline 2000 & 5.19 & 5.19 & 5.19 & 5.19 \\
\hline 3000 & 6 & 6.19 & 6.19 & 6.2 \\
\hline 4000 & 8.2 & 8.2 & 8.2 & 8.5 \\
\hline 5000 & 8.6 & 9.19 & 9.19 & 9.6 \\
\hline 5589 & 9 & 11 & 11.19 & 12 \\
\hline 6000 & 11.2 & 11.2 & 11.2 & \\
\hline 6354 & 11.25 & 11.2 & & \\
\hline 6780 & 11.3 & 12 & & \\
\hline 7000 & 11.52 & & & \\
\hline 8000 & 11.52 & & & \\
\hline 8523 & 12 & & & \\
\hline
\end{tabular}




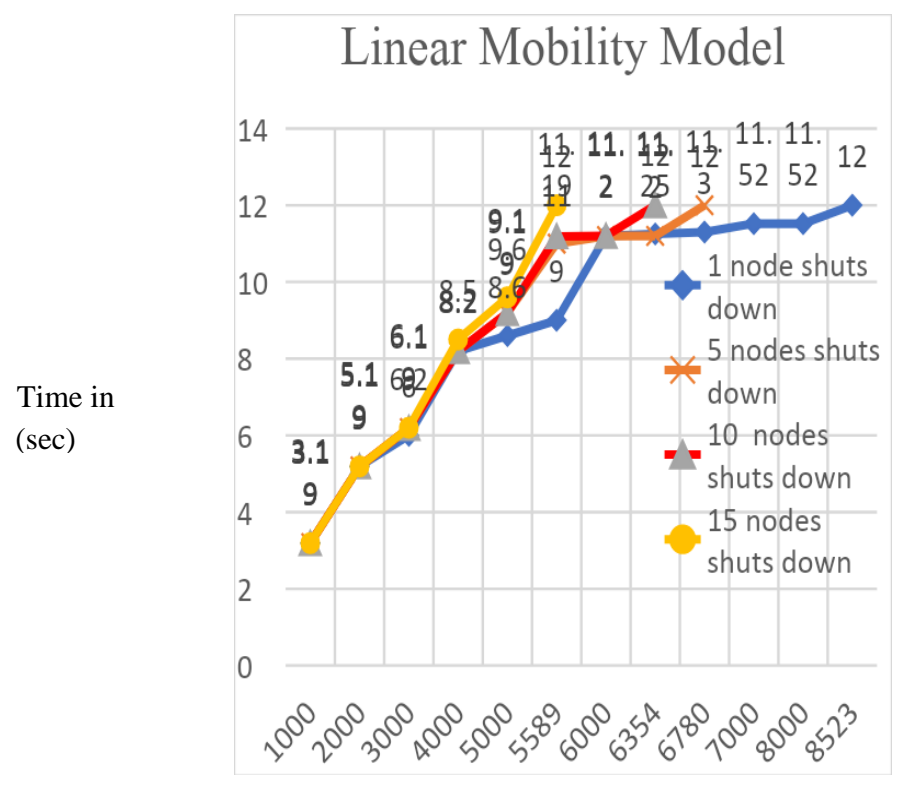

No. of Events

Fig. 3. Linear Deterministic Mod

Circle mobility mode: From table III it is known that when the all the nodes in a network are in Circle Mobility model the total number of events occurred at one node, five nodes, ten and fifteen nodes shutdown then total number of events are lesser when compared to Linear Deterministic Mobility model.

Table 3: Calculated values for Circle Deterministic model

\begin{tabular}{|c|c|c|c|c|}
\hline \multirow{3}{*}{ No of Events } & \multicolumn{4}{|c|}{ No of Nodes 20 } \\
\cline { 2 - 5 } & \multicolumn{4}{|c|}{ Time Taken When } \\
\cline { 2 - 5 } & 1 Node Shuts Down & $\begin{array}{c}\text { 5 Nodes Shuts } \\
\text { Down }\end{array}$ & $\begin{array}{c}\text { 10 Nodes Shuts } \\
\text { Down }\end{array}$ & $\begin{array}{c}15 \text { Nodes Shuts } \\
\text { Down }\end{array}$ \\
\hline 1000 & 3.19 & 3.19 & 3.19 & 3.19 \\
\hline 2000 & 4 & 4.19 & 4.19 & 4.4 \\
\hline 3000 & 6.8 & 7.19 & 7.19 & 7.6 \\
\hline 4000 & 9.2 & 9.19 & 9.2 & 9.6 \\
\hline 4642 & 9.2 & 9.5 & 10.19 & 12 \\
\hline 5000 & 9.2 & 10.3 & 11.1 & \\
\hline 5361 & 10 & 11.2 & 12 & \\
\hline 5558 & 10.5 & 12 & & \\
\hline 6000 & 11.19 & & & \\
\hline 6377 & 12 & & & \\
\hline
\end{tabular}




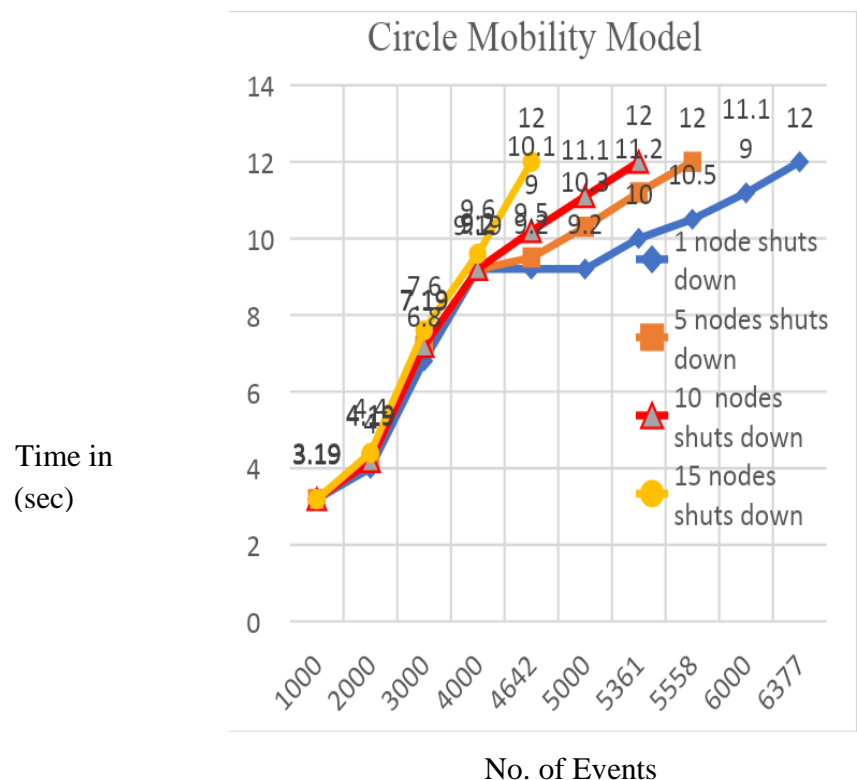

Fig. 4. Circle Deterministic Mobility Model

\subsection{Combining deterministic models}

Table 4: Calculated values for Deterministic model

\begin{tabular}{|c|c|c|c|c|}
\hline \multirow[t]{3}{*}{ No of Events } & \multicolumn{4}{|c|}{ No of Nodes 20} \\
\hline & \multicolumn{4}{|c|}{ Time Taken When } \\
\hline & 1 Node Shuts Down & $\begin{array}{c}5 \text { Nodes Shuts } \\
\text { Down }\end{array}$ & $\begin{array}{l}10 \text { Nodes Shuts } \\
\text { Down }\end{array}$ & $\begin{array}{l}15 \text { Nodes Shuts } \\
\text { Down }\end{array}$ \\
\hline 1000 & 3.2 & 3.2 & 3.2 & 3.2 \\
\hline 2000 & 5.8 & 6 & 6 & 6.19 \\
\hline 3000 & 6.5 & 6.62 & 6.6 & 6.5 \\
\hline 4000 & 7 & 7 & 7.01 & 8.5 \\
\hline 5000 & 7.01 & 7.4 & 8 & 10.8 \\
\hline 5273 & 7.09 & 8.01 & 8.3 & 12 \\
\hline 6000 & 7.19 & 9.52 & 10 & \\
\hline 7000 & 7.52 & 10 & 11.3 & \\
\hline 7154 & 7.54 & 10 & 12 & \\
\hline 8000 & 8.19 & 10.64 & & \\
\hline 8452 & 8.5 & 12 & & \\
\hline 9000 & 9.52 & & & \\
\hline 10000 & 10 & & & \\
\hline 11000 & 10.64 & & & \\
\hline 12000 & 11.6 & & & \\
\hline 12114 & 12 & & & \\
\hline
\end{tabular}




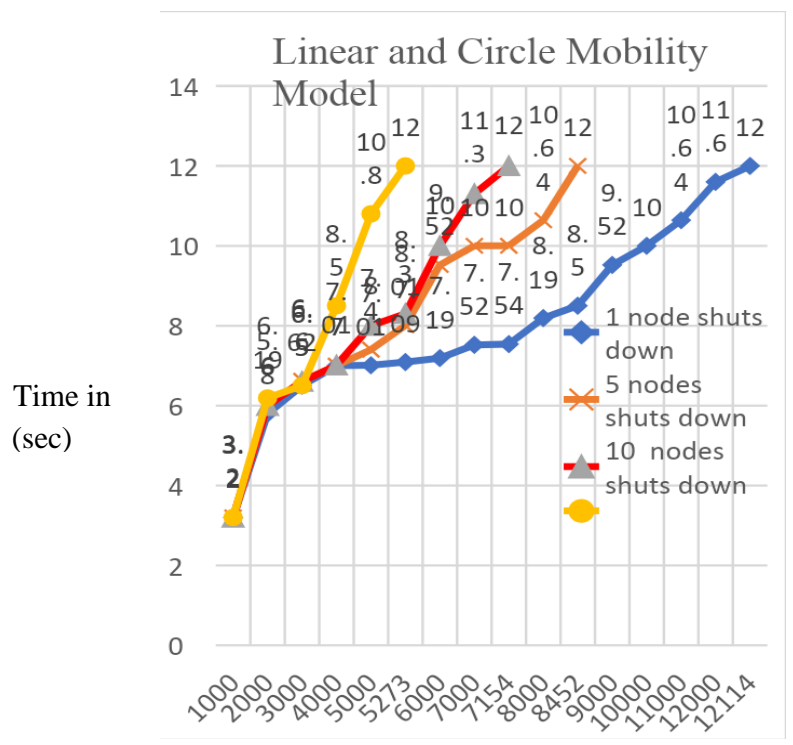

No. of Events

Fig. 5. Linear and Circle Deterministic Mobility Model

In table IV, the linear and Circle Deterministic Models are combined, there are 12114 events occurred by 12 seconds even after one node shutdown from the network. If five nodes, ten and fifteen nodes shutdown then total number of events are 8452, 7154 and 5273. By combining two Deterministic Models there is dramatic increase in number of events than the linear and circle models.

\subsection{Static and Deterministic model}

Table 5: Calculated for static and Mobility model

\begin{tabular}{|c|c|c|c|c|}
\hline \multirow{3}{*}{ No of Events } & \multicolumn{4}{|c|}{ No of Nodes 20 } \\
\cline { 2 - 5 } & \multicolumn{4}{|c|}{ Time Taken When } \\
\cline { 2 - 5 } & 1 Node Shuts Down & 5 Nodes Shut Down & $\begin{array}{c}10 \text { Nodes Shuts } \\
\text { Down }\end{array}$ & $\begin{array}{c}15 \text { Nodes Shut } \\
\text { Down }\end{array}$ \\
\hline 1000 & 3.2 & 3.2 & 3.2 & 3.2 \\
\hline 2000 & 3.52 & 3.52 & 3.52 & 3.52 \\
\hline 3000 & 3.99 & 3.99 & 3.99 & 3.53 \\
\hline 4000 & 4 & 4 & 4 & 5.7 \\
\hline 5000 & 4.6 & 4.64 & 4.64 & 8 \\
\hline 6000 & 5 & 5 & 5 & 10.3 \\
\hline 6704 & 5.8 & 5.19 & 5.19 & 12 \\
\hline 7000 & 6.1 & 5.21 & 5.21 & \\
\hline 8000 & 8.19 & 5.62 & 5.62 & \\
\hline 9000 & 10.3 & 6 & 6 & \\
\hline 9559 & 12 & 6.4 & 6.4 & \\
\hline
\end{tabular}




\begin{tabular}{|l|l|c|c|l|}
\hline 10000 & & 6.52 & 6.5 & \\
\hline 11000 & & 6.64 & 6.64 & \\
\hline 12000 & & 7.52 & 7.52 & \\
\hline 13000 & & 9.2 & 9.5 & \\
\hline 14000 & & 11.19 & 11.5 & \\
\hline 14594 & & 11.52 & 12 & \\
\hline 15000 & & 11.52 & & \\
\hline 15721 & & 12 & & \\
\hline
\end{tabular}

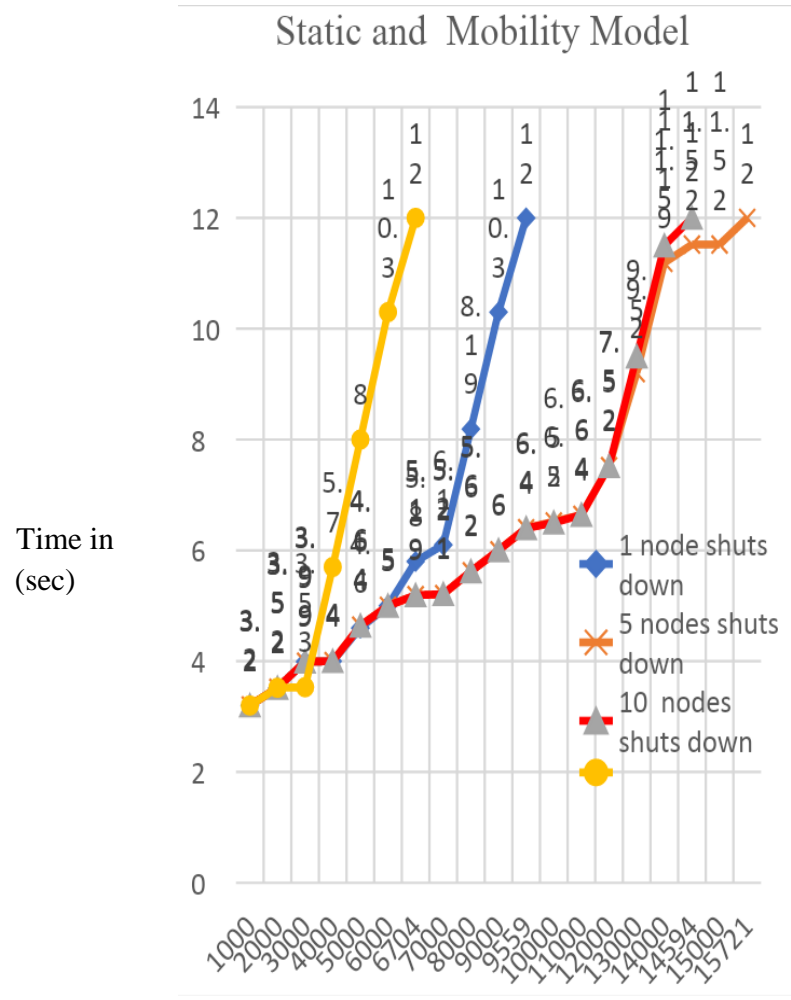

No. of Events

Fig. 6. Static and Mobility Model

In table V, the Stationary and Deterministic Models are combined, there are 9559 events occurred by 12 seconds even after one node shutdown from the network. The source and destination node are in stationary model and all other eighteen nodes are in Circular Deterministic Models. If five nodes, ten and fifteen nodes shutdown then total number of events are 15721, 14594 and 6704. By combining two Different AODV Models there is dramatic increase in number of events during five and ten nodes shutdown than one node shutdown. But there is decrease in number of events for fifteen nodes shutdown when compared to one node shut down. 


\section{Conclusion}

In this paper the analysis of performance of Stationary model and Deterministic Mobility models are done using AODV reactive routing protocol. The simulation is carried out in OMNET++ Simulator and the results are presented. After the analysis, we observed that combining Stationary and Deterministic model gives 20\% higher performance when compared to other models in terms of number of events and time for more than one node shutdown. By combining two Deterministic Models gives $10 \%$ higher performance when compared to other models in terms of number of events and time for one node shutdown. The work can be extended for detecting the black hole attack by finding the average end to end delay, packet delivery ratio and throughput.

\section{$5 \quad$ References}

[1] Sabih ur Rehman, M. Arif Khan, Tanveer A. Zia, Lihong Zheng," Vehicular Ad-Hoc Networks (VANETs) - An Overview and Challenges". Journal of Wireless Networking and Communications 2013, doi: 10.5923/ jjwnc.20130303.02, pp 3(3): 29-38.

[2] Anna Maria Vegni, Mauro Biagi and Roberto Cusani," Smart Vehicles, Technologies and Main Applications in Vehicular Ad hoc Networks". INTECH Open Science |Open Minds https://doi.org/10.5772/55492

[3] M.Danya Priyadharshini, Christo Ananth," A Secure Hash Message Authentication Code to Avoid Certificate Revocation List Checking In Vehicular Adhoc Networks". International Journal of Applied Engineering Research, ISSN 0973-4562 Vol. 10 No.2 (2015), pp. 1250-1254.

[4] Vinita Jindal, Punam Bedi," Vehicular Ad-Hoc Networks: Introduction, Standards, Routing Protocols and Challenges". IJCSI International Journal of Computer Science Issues, Volume 13, Issue 2, March 2016 ISSN (Print): 1694-0814 | ISSN (Online): 16940784, https://doi.org/10.20943/01201602.4455.

[5] Manpreet kaur, Nitin Bhagat," A Review on Various Attacks in VANET". IJCAT International Journal of Computing and Technology, Volume 3, Issue 5, May 2016, ISSN: 2348 - 6090, pp:292-294.

[6] I NET Framework, https://inet.omnetpp.org/docs/ users-guide/ch-introduction.html\#whatis-inet-framework.

[7] OMNET ++ Simulation Manual, Https://doc.omnetpp.org/omnetpp/manual/.

[8] Node Mobility, Https://inet.omnetpp.org/docs/users-guide/ch-mobility.html

[9] Arvind Kumar Shukla, C.K.Jha, Nitin Sexena, Sanjay Kumar Biswash," The analysis of AODV, based on mobility model". $3^{\text {rd }}$ IEEE International Advance Computing Conference (IACC), doi:10.1109/iadcc.2013.6514266, 2013, pp:440-443. https://doi.org/10.1109/ iadcc.2013.6514266

[10] Prashant Kumar Maurya, Gaurav Sharma, Vaishali Sahu, Ashish Roberts, Mahendra Srivastava," An Overview of AODV Routing Protocol". International Journal of Modern Engineering Research (IJMER), vol.2, Issue.3, May-June 2012, ISSN: 2249-6645, pp:728732.

[11] Halabi Hasbullah, Irshad Ahmed Soomro, Jamalul-lail Ab Manan," Denial of Service (DOS) Attack and Its Possible Solutions in VANET". World Academy of Science, 
Engineering and Technology International Journal of Electronics and Communication Engineering, vol:4 No.5,2010, pp:813-817.

[12] Durga R, Poovammal E.," Generation of RAESSES Hash Function for Medical Blockchain Formation Based on High Dynamic Chaotic Systems." International Journal of Advanced Science and Technology, Issue Vol. 29 No. 06 (2020), 29(06), 8427-8440.

[13] Mohammad Alnabhan, Mahmoud Alshuqran, Mustafa Hammad, Mohammad Al Nawayseh, "Performance Evaluation of Unicast Routing Protocols in MANETs - Current State and Future Prospects". International Journal of Interactive Mobile Technologies (iJIM), Vol:11 No.01, https://doi.org/10.3991/ijim.v11i1.6295

[14] Mada' Abdel Jawad, Saeed Salah, Raid Zaghal," DSDV Extension to Enhance the Performance of Ad Hoc Networks in High Diverse-Velocity Environments". International Journal of Interactive Mobile Technologies (iJIM), Vol: 14 No.06, https://doi.org/10. 3991/ijim.v14i06.11889.

[15] Petros Mashwama, Stephen Gbenga Fashoto, Elliot Mbunge, Simanga Gwebu, "Development of a Mobile Inter-Vehicular Communication System Based on Gossip Algorithm". International Journal of Interactive Mobile Technologies (iJIM), Vol:14 No.11, https://doi.org/10.3991/ijim.v14i11.12949

\section{Authors}

G. Kothai is a Research Scholar in the Department of Computer Science and Engineering at SRM Institute of Science and Technology. She obtained her B.E. Degree in Computer Science and Engineering at Sasurie Academy of Engineering in the year 2016 and M.E degree in Computer Science and Engineering from Coimbatore Institute of Technology. Her research interests include Vehicular adhoc network security, Cyber Security, Network security and Machine Learning. Email: kothaig@srmist.edu.in

Dr. E. Poovammal is a Professor in the Department of Computer Science and Engineering at SRM Institute of Science and Technology. She joined in SRM in the year 1996. Before joining SRM, she worked in industry for five years. She obtained her B.E. Degree in Electrical and Electronics Engineering from Madurai Kamaraj University in the year 1990, M.E degree in Computer Science and Engineering from Madras University and Ph.D. degree in Computer Science and Engineering from SRM University. Her research interests include Big Data Analytics and Machine Learning. She is certified as Adjunct Faculty by Institute of software Research, Carnegie Mellon University, Pittsburgh, USA and served for 4 years. She has published more than 40 referred journals and presented in various international and national conferences. She is fellow member of IE (I) and active member of professional bodies such as IEEE, IET, ACM, ISCA, ISTE. Email: poovamme@srmist.edu.in

Article submitted 2020-06-26. Resubmitted 2020-07-25. Final acceptance 2020-07-26. Final version published as submitted by the authors. 